\title{
A NEW ANALYTIC SYMPLECTIC ELASTICITY APPROACH FOR BEAMS RESTING ON PASTERNAK ELASTIC FOUNDATIONS
}

\author{
C. F. LÜ, C. W. LIM AND W. A. YAO
}

\begin{abstract}
Analytic solutions describing the stresses and displacements of beams on a Pasternak elastic foundation are presented using a symplectic method based on classical two-dimensional elasticity theory. Hamilton's principle with a Legendre transformation is employed to derive the Hamiltonian dual equation, and separation of variables reduces the dual equation to an eigenequation that differs from the conventional eigenvalue problems involved in vibration and buckling analysis. Using adjoint symplectic orthonormality, a group of eigensolutions of zero eigenvalue, corresponding to the Saint-Venant problem, are derived. This approach differs from the traditional semi-inverse analysis, which requires stress or deformation trial functions in the Lagrangian system. The final solutions, which account for the effects of an elastic foundation and applied lateral loads, are approximated by an eigenfunction expansion. Comparisons with existing numerical solutions are conducted to validate the efficiency of this new approach.
\end{abstract}

\section{Introduction}

Isotropic beams and plates on elastic foundations are widely used to model civil engineering structures, including building footings designed in the form of plates or planar framed structures, bases of artificial navigable waterways, the pavement of highways and runways, and rails, to name a few. Much of the existing literature on structure-foundation systems is based on classical thin beam/plate theory [Leissa 1973; Franciosi and Masi 1993; De Rosa and Maurizi 1998], which relies on the assumption that a foundation's reaction forces act on the midplane of the modeled beam/plate. This assumption, however, may be unrealistic for thicker structures in which shear deformations may be significant. In addition, the effects of the foundation on deformation and stress fields in the vicinity of the two lateral surfaces become important for larger beam and plate aspect ratios.

A variety of first-order shear deformation theories [Timoshenko 1921; Shirima and Giger 1992; Wang et al. 1998; Lee et al. 2003] and refined higher-order theories [Reddy 1984; Frostig et al. 1992; Matsunaga 2000], which account for shear deformations and/or rotary inertia, have been proposed to improve the accuracy of thick beam/plate models. However, they suffer from the shortcoming that transverse normal stress is neglected [Lim 1999].

A number of studies have attempted to present two-dimensional elasticity solutions for isotropic and anisotropic beams. Whitney [1985] performed a stress analysis on orthotropic beams subjected to concentrated loads within the framework of the classical theory of elasticity. Stresses that developed during interlaminar beam tests were examined in detail, and observations showed considerable deviation from

Keywords: Saint-Venant problem, elastic foundation, symplectic, Hamilton principle, Legendre transformation.

This work was supported by Research Scholarship Enhancement Scheme of City University of Hong Kong, and Project No. 7002291 (BC). 
classical beam theory over large portions of the beam. Sullivan and Vanoene [1986] derived an elasticity solution for centrally loaded orthotropic beams by virtue of a stress function and the Fourier series. The symmetric properties of the stress field in the beams were investigated and compared to those predicted by beam theory. Regions in which the beam theory predictions coincided with the theory of elasticity were identified. Sankar [2001] reported an exact elasticity solution for a simply supported functionally graded beam with the aid of the Fourier series. Although no assumptions concerning the stress and deformation fields were adopted in these papers, and, hence, the solution formulations were applicable to beams of arbitrary thickness, such exact solutions are only available for fully simply supported beams. Ding et al. [2005] proposed analytic elasticity solutions for the stress and displacement fields of an isotropic fixedfixed beam produced by uniform loading. They constructed biharmonic stress functions using the Airy stress function method, and investigated the differences between the two types of fixed ends described in [Timoshenko and Goodier 1970]. Their analysis employed the semi-inverse method, which is the approach of choice for dealing with a higher-order partial differential equation with a single variable. Such single-variable solution procedures rely on the Lagrangian method, for which many effective methods of mathematical physics, such as separation of variables and expansion of eigenfunctions, are not applicable.

We have not been able to find in the literature analytic solutions for generally supported beams resting on elastic foundations using classical two-dimensional theory of elasticity. In this paper, a rational derivation based on that theory is presented to determine the mechanical behavior of such beams. The solution involves the symplectic group, which was introduced by Weyl [1939] and has found applications in many areas of physics, including quantum mechanics, relativity, gravitation, astrophysics, classical mechanics, Hamiltonian mechanics, and elasticity. Details regarding the development and applications of the symplectic approach can be found in [Lim et al. 2007].

In the Hamiltonian framework, separation of variables and eigenfunction expansions can be applied to derive exact analytic solutions for some basic elasticity problems, although solutions are still unavailable and have long been a bottleneck for the development of the theory of elasticity. In [Lim et al. 2008] we proposed a new symplectic approach to analyze the bending behavior of corner-supported thin plates subjected to uniform transverse pressures, for which an exact explicit solution for deflection is derived here for the first time. The zero bending moment and shear forces at the free edges are exactly satisfied, and the twisting moment conditions at the support corners are exactly predicted. These quantities have long eluded both analytic and numerical analysis, including application of the finite element method.

For beams in a plane state without body forces, a Legendre transformation may be applied to derive the conservative Hamiltonian variational principle, from which a Hamiltonian dual equation, i.e., the symplectic dual system, is formulated. With homogeneous side boundary conditions, the method of separation of variables is employed to obtain the eigenequation for the transverse cross section. The present work emphasizes eigensolutions of zero eigenvalue, because they correspond to the basic solutions of Saint-Venant problems [Yao et al. 2009].

The effect of an elastic foundation on the mechanical behavior of a beam arises from the interaction between the foundation and the beam surface, which are unlike those modeled in classical beam theory. Hence, an elastic foundation is treated with side boundary conditions, similar to those of an applied load, and the contributions of these boundary conditions to the solutions for beams are approximated by a linear expansion of all eigensolutions of zero eigenvalue. A comparison of numerical examples with other methods is presented to illustrate the accuracy of the present symplectic approach. 


\section{Hamilton's variational principle and symplectic formulation}

Consider an isotropic beam of length $l$, depth $2 h$, and unit width. The Cartesian coordinate system is defined such that $-h \leq z \leq h$ and $0 \leq x \leq l(l \gg 2 h)$, as shown in Figure 1. Suppose that all loads (including the boundary conditions) are applied in the $x O z$ plane and remain constant along the width. These conditions define a plane stress problem involving a beam.

Here, the top surface of the beam is subjected to the following applied loads:

$$
\sigma_{z}=\bar{F}_{z 1}(x), \quad \tau_{x z}=\bar{F}_{x 1}(x), \quad \text { at } z=-h,
$$

and the bottom surface is attached to an elastic foundation (see Figure 1). We assume that the foundation experiences only vertical displacements without horizontal movement, and that only the compatibility of normal displacements at the foundation-beam interface is considered. Hence, the beam is subjected to normal reaction forces by the foundation, which is modeled as a two-parameter foundation. The displacement-reaction force relation [Pasternak 1954] is

$$
\sigma_{z}=\bar{F}_{z 2}(x)=k_{p} \frac{\partial^{2} w}{\partial x^{2}}-k_{w} w, \quad \tau_{x z}=\bar{F}_{x 2}(x)=0, \quad \text { at } z=h,
$$

where $k_{w}$ is the normal stiffness (modulus of the Winkler foundation) and $k_{p}$ the shear stiffness of the foundation.

For an isotropic beam in a plane stress state as described above, the constitutive equations are

$$
\left\{\begin{array}{c}
\sigma_{x} \\
\sigma_{z} \\
\tau_{x z}
\end{array}\right\}=\frac{E}{1-v^{2}}\left[\begin{array}{ccc}
1 & v & 0 \\
v & 1 & 0 \\
0 & 0 & \frac{1}{2}(1-v)
\end{array}\right]\left\{\begin{array}{c}
\frac{\partial u}{\partial x} \\
\frac{\partial w}{\partial z} \\
\frac{\partial w}{\partial x}+\frac{\partial u}{\partial z}
\end{array}\right\},
$$

where $\sigma_{x}$ and $\sigma_{z}$ are the normal stresses, $\tau_{x z}$ is the shear stress, $u$ and $w$ are the displacement components, respectively, in the $x$ - and $z$-directions, $E$ and $v$ are the Young's modulus and Poisson's ratio, respectively. In the absence of body forces, the governing differential equations of the beam in equilibrium are

$$
\frac{\partial \sigma_{x}}{\partial x}+\frac{\partial \tau_{x z}}{\partial z}=0, \quad \frac{\partial \tau_{x z}}{\partial x}+\frac{\partial \sigma_{z}}{\partial z}=0
$$
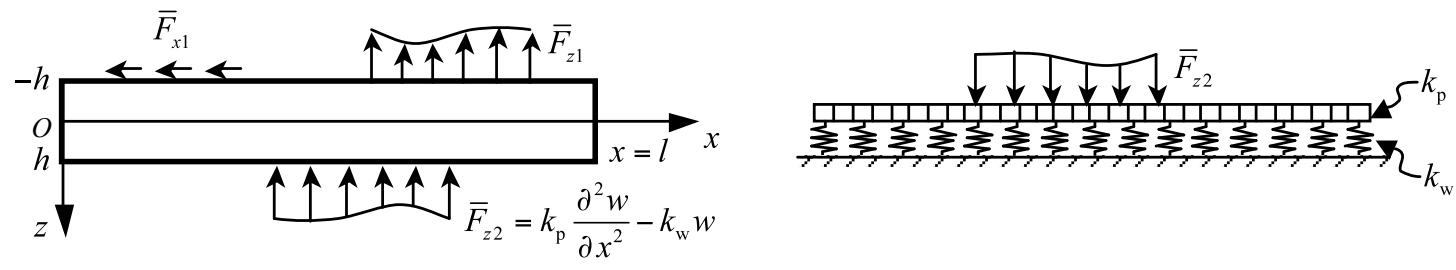

Figure 1. Left: geometry, coordinate system and loading condition of a beam resting on elastic foundations. Right: Pasternak foundation and beam foundation. 
In the following Hamiltonian formulation, the time variable is designated as the $x$ coordinate, and a dot indicates partial differentiation with respect to $x$. Following the routine transformation of the Lagrangian system into the Hamiltonian system, via the Legendre transformation, we arrive at the Hamiltonian variational principle,

$$
\delta\left(\int_{0}^{l} \int_{-h}^{h}\left[\boldsymbol{p}^{T} \dot{\boldsymbol{q}}-\mathscr{H}(\boldsymbol{q}, \boldsymbol{p})\right] d x d z+\int_{0}^{l}\left(\overline{\boldsymbol{F}}_{1}^{T} \boldsymbol{q}\right)_{z=-h} d x+\frac{1}{2} \int_{0}^{l}\left(k_{w} w^{2}+k_{p} \dot{w}^{2}\right)_{z=h} d x\right)=0,
$$

where $\mathscr{H}(\boldsymbol{q}, \boldsymbol{p})$ is the Hamiltonian energy density, as given in [Yao et al. 2009], $\boldsymbol{q}=\left[\begin{array}{ll}u & w\end{array}\right]^{T}$ and $\boldsymbol{p}=\left[\begin{array}{ll}\sigma & \tau\end{array}\right]^{T}$ (with $\sigma=\sigma_{x}$ and $\tau=\tau_{x z}$ ) are a pair of dual vectors, and $\overline{\boldsymbol{F}}_{1}^{T}=\left[\bar{F}_{x 1} \bar{F}_{z 1}\right]$. Based on (5), the Hamiltonian dual equation is derived as

$$
\dot{\boldsymbol{v}}=\boldsymbol{H} \boldsymbol{v}
$$

where $\boldsymbol{v}=\left[\begin{array}{llll}u & w & \sigma & \tau\end{array}\right]^{T}$ is called the state vector, and $\boldsymbol{H}$ is the Hamiltonian operator matrix [Yao et al. 2009]. From (3), the induced variable $\sigma_{z}$ is obtained as

$$
\sigma_{z}=E \frac{\partial w}{\partial z}+v \sigma
$$

For the present Hamiltonian dual equation (6), the method of variable separation is applicable, i.e., the solution to (6) is assumed to be of the form

$$
\boldsymbol{v}(x, z)=\xi(x) \psi(z) .
$$

Substitution of (8) into (6) yields

$$
\xi(x)=e^{\mu x},
$$

where $\mu$ is the eigenvalue in the $x$-direction, and the eigenvalue equation

$$
\boldsymbol{H} \boldsymbol{\psi}(z)=\mu \boldsymbol{\psi}(z)
$$

where $\psi(z)$ is the eigenvector, which fulfills the homogeneous boundary conditions

$$
E \frac{\partial w}{\partial z}+v \sigma=0, \quad \tau=0, \quad \text { at } z= \pm h .
$$

\section{Basic solutions to the Saint-Venant problem}

Basic solutions. According to the Saint-Venant principle, forces in equilibrium pose local influences, i.e., local effects decay dramatically with the distance. The solution to the Saint-Venant problem is inherent in the eigensolutions with nonzero eigenvalues. Meanwhile, (8) implies that the zero eigenvalue solutions are not sensitive to the equilibrium system of forces, because no exponential functions are present. Hence, for the slender beam $(l \gg 2 h)$, the effects of the equilibrium system of forces at the two ends are negligible, that is, the eigensolutions of nonzero eigenvalues can be excluded. As a result, only eigensolutions for the repeated zero-valued eigenvalue $\mu=0$ will be used to construct the basic solution to the beam in the Saint-Venant problem [Yao et al. 2009], i.e.,

$$
\boldsymbol{v}=a_{1} \boldsymbol{\psi}_{f}^{(0)}+a_{2} \boldsymbol{\psi}_{f}^{(1)}+a_{3} \boldsymbol{\psi}_{s}^{(0)}+a_{4} \boldsymbol{\psi}_{s}^{(1)}+a_{5} \boldsymbol{\psi}_{s}^{(2)}+a_{6} \boldsymbol{\psi}_{s}^{(3)},
$$


where $\boldsymbol{\psi}_{f}^{(n)}$ and $\boldsymbol{\psi}_{s}^{(n)}$ are, respectively, the Jordan form eigensolutions of the first and second chains, for which explicit expressions have been derived in [Yao et al. 2009]. Equation (12) can be expanded to

$$
\begin{array}{ll}
u=a_{1}-a_{4} z+a_{6}\left(-\frac{6+5 v}{10} h^{2} z+\frac{2+v}{6} z^{3}\right), & \sigma=a_{2} E-a_{5} E z, \\
w=-a_{2} v z+a_{3}-a_{5}\left(\frac{4+5 v}{10} z^{2}-\frac{v}{2} z^{2}\right), & \tau=\frac{1}{2} a_{6} E\left(z^{2}-h^{2}\right),
\end{array}
$$

where the $a_{i}$ are $x$-dependent parameters to be determined using the constraints at $x=0, l$. The basic solution in (13) is applicable to the Hamiltonian dual equation (6) with the homogeneous side boundary conditions given in (11). When the beam is subjected to external loading and elastic foundations, $a_{i}$ should be determined from the Hamiltonian variational principle in (5).

Determination of the $\boldsymbol{a}_{i}$. Because each basic eigensolution in (12) is related to a special deformation, the $a_{i}$ should be related to particular physical quantities. Here, $a_{1}, a_{3}, a_{4}$ correspond to axial deformation, transverse deformation, and rotation angle, respectively, and $a_{2}, a_{5}, a_{6}$ correspond to axial force, bending moment, and shear force, respectively. Note that $a_{1}$ and $a_{2}$ are related to symmetric deformations, whereas $a_{3}-a_{6}$ are related to antisymmetric deformations.

In the present analysis, only the bending behavior of the beam on an elastic foundation is considered. The symmetric deformations described by $a_{1}$ and $a_{2}$ are therefore excluded. Substituting the antisymmetric portion of (13) into (5) leads to

$$
\begin{aligned}
& \dot{a}_{3}=a_{4}+\frac{4+5 v}{10 E} \bar{F}_{x 1}, \quad \dot{a}_{4}=a_{5}-0.4 h^{2} \dot{a}_{6}, \quad \dot{a}_{5}=a_{6}+\frac{1}{E I} \bar{F}_{x 1}, \\
& \dot{a}_{6}=-\frac{1}{E I}\left(k_{w}\left(a_{3}-0.4 h^{2} a_{5}\right)-k_{p}\left(\ddot{a}_{3}-0.4 h^{2} \ddot{a}_{5}\right)+\bar{F}_{z 1}\right),
\end{aligned}
$$

where $I=\frac{2}{3} h^{3}$ is the second moment of the area. The set of coupled differential equations above can be reduced to a fourth-order differential equation with respect to $a_{3}$, i.e.,

$$
\bar{E} a_{3}^{(4)}-\bar{k}_{p} a_{3}^{(2)}+k_{w} a_{3}=f(x),
$$

where $f(x)=\left(\left(E I+0.4 k_{p} h^{2}\right) g-0.4 h^{3}\right) \dddot{\vec{F}}_{x 1}+\left(h-0.4 g k_{w} h^{2}\right) \dot{\bar{F}}_{x 1}+0.4 h^{2} \ddot{\bar{F}}_{z 1}-\bar{F}_{z 1}, \quad \bar{E}=E I+0.8 k_{p} h^{2}$, $\bar{k}_{p}=k_{p}+0.8 k_{w} h^{2}$, and $g=(2+v) /(2 E)$. The eigenvalues of (15) are

$$
\pm r_{1}=\sqrt{\frac{\bar{k}_{p}+\sqrt{\bar{k}_{p}^{2}-4 \bar{E} k_{w}}}{2 \bar{E}}}, \quad \pm r_{2}=\sqrt{\frac{\bar{k}_{p}-\sqrt{\bar{k}_{p}^{2}-4 \bar{E} k_{w}}}{2 \bar{E}}} .
$$

We find that $r_{2}=0$ when $k_{w}=0$, and $r_{1}=r_{2}=0$ when $k_{w}=k_{p}=0$. Hence, the general solution, $a_{3}$, depends on whether $k_{w}$ and $k_{p}$ vanish:

$$
\begin{array}{ll}
\text { Case 1: } k_{w} \neq 0 & a_{3}=c_{1} e^{r_{1} x}+c_{2} e^{-r_{1} x}+c_{3} e^{r_{2} x}+c_{4} e^{-r_{2} x}+\tilde{a}_{3} ; \\
\text { Case 2: } k_{w}=0, k_{p} \neq 0 & a_{3}=c_{1} e^{r_{1} x}+c_{2} e^{-r_{1} x}+c_{3}+c_{4} x+\tilde{a}_{3} ; \\
\text { Case 3: } k_{w}=0, k_{p}=0 & a_{3}=c_{1}+c_{2} x+c_{3} x^{2}+c_{4} x^{3}+\tilde{a}_{3} .
\end{array}
$$

Here, the $c_{i}$ are constants of integration and $\tilde{a}_{3}$ is the particular solution to (15) for the inhomogeneous term $f(x)$ for the applied loads. 
Knowing the values for $a_{3}$, all remaining parameters $a_{4}-a_{6}$ can be determined, based on (14):

$$
\begin{aligned}
& a_{4}=\dot{a}_{3}-\frac{4+5 v}{10 E} \bar{F}_{x 1}, \\
& a_{5}=\frac{1}{\tilde{E}}\left(\bar{E} \ddot{a}_{3}-0.4 k_{w} h^{2} a_{3}+g_{1} \dot{\bar{F}}_{x 1}-0.4 h^{2} \bar{F}_{z 1}\right), \\
& a_{6}=\frac{1}{\tilde{E}}\left(\bar{E} \dddot{a}_{3}-0.4 k_{w} h^{2} \dot{a}_{3}+g_{1} \ddot{\bar{F}}_{x 1}-0.4 h^{2} \dot{\bar{F}}_{z 1}\right)-\frac{h}{E I} \bar{F}_{x 1},
\end{aligned}
$$

where $\tilde{E}=E I+0.4 h^{2}\left(k_{p}-0.4 k_{w} h^{2}\right)$ and $g_{1}=0.4 h^{3}-g\left(E I+0.4 k_{p} h^{2}\right)$.

In practice, the constants in Equations (17) should be determined according to the support conditions at the two ends. Considering the energy related to the end constraints in accordance with the Hamiltonian variational principle in (5), the following three typical constraint conditions at $x=0$ and $x=l$, for clamped (C), simple supports $(\mathrm{S})$, and free $(\mathrm{F})$ supports, are derived, where $\lambda=0.4(1+v) h^{2}$ :

$$
\begin{array}{ll}
\mathrm{F}: & a_{5}=a_{6}=0 \\
\mathrm{~S}: & a_{3}=a_{5}=0 \\
\mathrm{C}: & a_{3}-\lambda a_{5}=0, \quad a_{4}+\lambda a_{6}=0
\end{array}
$$

According to the previously described interpretations of the $a_{i}$, the expression in (21) can be interpreted physically as a case of zero axial force, zero bending moment, and zero shear force, while the expression in (22) can be interpreted as zero axial force, zero transverse displacement, and zero bending moment. Similarly, in (23), the first condition represents an axial displacement of zero for the clamped end, but the remaining two expressions indicate a zero equivalent transverse displacement and zero equivalent rotation angle, respectively [Yao et al. 2009].

\section{Bending of beams due to uniform pressure}

To illustrate applications of the present symplectic approach, a beam subjected to a uniform pressure on the top surface is considered. The applied loading conditions in (1) are expressed as

$$
\bar{F}_{x 1}=0, \quad \bar{F}_{z 1}=-q_{0}, \quad \text { at } z=-h,
$$

where $p_{0}$ is a constant independent of $x$. Substituting these conditions into (15) yields

$$
\left(E I+0.8 k_{p} h^{2}\right) a_{3}^{(4)}-\left(k_{p}+0.8 k_{w} h^{2}\right) a_{3}^{(2)}+k_{w} a_{3}=q_{0},
$$

for which a particular solution should be sought according to the foundation parameters. Combining (17)-(21) with (25) leads to the general solutions for $a_{3}-a_{6}$ :

$$
\text { Case 1: } k_{w} \neq 0 \quad\left\{\begin{array}{l}
a_{3}=c_{1} e^{r_{1} x}+c_{2} e^{-r_{1} x}+c_{3} e^{r_{2} x}+c_{4} e^{-r_{2} x}+q_{0} / k_{w}, \\
a_{4}=c_{1} r_{1} e^{r_{1} x}-c_{2} r_{1} e^{-r_{1} x}+c_{3} r_{2} e^{r_{2} x}-c_{4} r_{2} e^{-r_{2} x} \\
a_{5}=\frac{1}{\tilde{E}}\left(c_{1} M_{1} e^{r_{1} x}+c_{2} M_{1} e^{-r_{1} x}+c_{3} M_{2} e^{r_{2} x}+c_{4} M_{2} e^{-r_{2} x}\right), \\
a_{6}=\frac{1}{\tilde{E}}\left(c_{1} M_{1} r_{1} e^{x}-c_{2} M_{1} r_{1} e^{-r_{1} x}+c_{3} M_{2} r_{2} e^{r_{2} x}-c_{4} M_{2} r_{2} e^{-r_{2} x}\right)
\end{array}\right.
$$




$$
\begin{aligned}
& \text { Case 2: } k_{w}=0, k_{p} \neq 0 \quad\left\{\begin{array}{l}
a_{3}=c_{1} e^{r_{1} x}+c_{2} e^{-r_{1} x}+c_{3}+c_{4} x-q_{0} x^{2} / 2 k_{p}, \\
a_{4}=c_{1} r_{1} e^{r_{1} x}-c_{2} r_{1} e^{-r_{1} x}+c_{4}-q_{0} x / k_{p}, \\
a_{5}=\frac{1}{\tilde{E}} M_{1}\left(c_{1} e^{r_{1} x}+c_{2} e^{-r_{1} x}\right)+\frac{q_{0}}{\tilde{E}}\left(0.4 h^{2}-\bar{E} / k_{p}\right), \\
a_{6}=\frac{1}{\tilde{E}} M_{1} r_{1}\left(c_{1} e^{r_{1} x}-c_{2} e^{-r_{1} x}\right) ;
\end{array}\right. \\
& \text { Case 2: } k_{w}=0, k_{p}=0 \quad\left\{\begin{array}{l}
a_{3}=c_{1}+c_{2} x+c_{3} x^{2}+c_{4} x^{3}+\frac{q_{0}}{24 E I} x^{4} \\
a_{4}=c_{2}+2 c_{3} x+3 c_{4} x^{2}+\frac{q_{0}}{6 E I} x^{3}, \\
a_{5}=2 c_{3}+6 c_{4} x+\frac{q_{0}}{2 E I} x^{2}+\frac{6 q_{0}}{5 E A}, \\
a_{6}=6 c_{4}+\frac{q_{0}}{E I} x
\end{array}\right.
\end{aligned}
$$

where $M_{1}=\bar{E} r_{1}^{2}-0.4 k_{w} h^{2}$ and $M_{2}=\bar{E} r_{2}^{2}-0.4 k_{w} h^{2}$.

In this paper, three combinations of the typical end constraints in (21)-(23) are considered: clampedclamped (CC), simply supported at both ends (SS), and clamped-free (CF). Incorporating these constraints into the three cases describing elastic foundations gives rise to an algebraic matrix equation governing the integral constants $c_{i}$ :

$$
D c=p,
$$

where $\boldsymbol{c}=\left[\begin{array}{llll}c_{1} & c_{2} & c_{3} & c_{4}\end{array}\right]^{T}$. The explicit expressions of the coefficient matrix $\boldsymbol{D}$ and vector $\boldsymbol{p}$ for each beam in Cases 1 and 2 are given in the Appendix. Case 3 is exactly the case without an elastic foundation, for which the coefficients are simple and can be expressed explicitly. Hence the parameters $a_{3}-a_{6}$ are directly presented in the Appendix. From (29), the $c_{i}$ are obtained as

$$
c_{i}=\sum_{k=1}^{4} \frac{d_{k i}}{D} p_{i}
$$

where $d_{k i}=(-1)^{k+i}\left|\overline{\boldsymbol{D}}_{k i}\right|(i=1,2,3,4)$ is the algebraic complement of the element $D_{k i}, \overline{\boldsymbol{D}}_{k i}$ is obtained by eliminating the $k$ th row and $i$ th column of $\boldsymbol{D}$, and $p_{i}$ is the element of $\boldsymbol{p}$.

\section{Numerical comparisons and discussions}

To validate the rationality, accuracy, and effectiveness of this symplectic elasticity approach, comparisons are presented for slender beams $l \gg 2 h$ on an elastic foundation. For generality and brevity, the following nondimensional parameters and variables are introduced:

$$
(U, W)=\frac{(u, w) E I}{q_{0} l^{4}}, \quad X=\frac{\sigma_{x}}{q_{0}}, \quad K_{w}=\frac{k_{w} l^{4}}{E I}, \quad K_{p}=\frac{k_{p} l^{2}}{E I} .
$$

In all examples, the Poisson's ratio is taken as $v=0.3$.

Bending of beams without elastic foundation. As a first attempt, the bending behavior of beams without elastic foundations $\left(K_{w}=K_{p}=0\right)$ is considered, and the solutions are compared with results from other methods. Figure 2 shows the longitudinal distributions of nondimensional transverse displacements $W(x, 0)$ at the neutral line and the axial normal stress $X(x, h)$ at the lower surface of CC beams with different aspect ratios. The figures show that the present results (solid lines) agree well with the results obtained using the semianalytic method (dashed lines) in [Chen et al. 2004] based on two-dimensional 

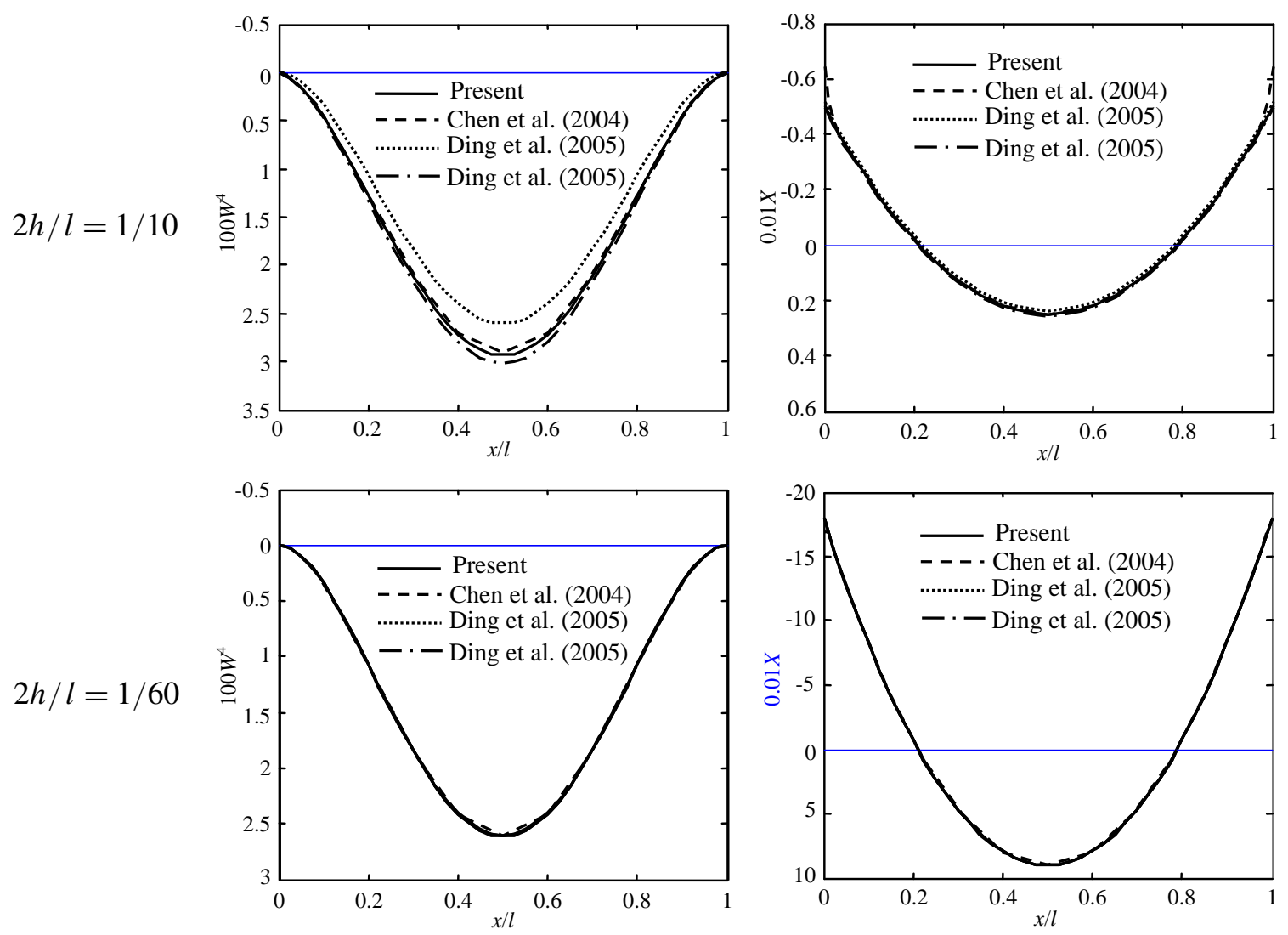

Figure 2. Deflection curves $W(x, 0)$ and longitudinal axial normal stresses $X(x, h)$ for CC beams without elastic foundations $\left(K_{w}=K_{p}=0\right)$.

elasticity theory without invoking assumptions regarding the deformations and stresses. We emphasize a comparison of these results with those of Ding et al. [2005], who used the Airy stress function based on two-dimensional elasticity theory. They considered two kinds of constraint conditions for clamped ends, as described in [Timoshenko and Goodier 1970]:

$$
\frac{\partial w}{\partial x}=0 \quad \text { and } \quad \frac{\partial u}{\partial z}=0
$$

Figure 2 illustrates the results corresponding to these two end conditions, with dotted lines for the first condition and dash-dot lines for the second. For the case $2 h / l=1 / 10$, the present result is significantly larger than the analytic solution for the first type of clamped end, but is somewhat smaller than the analytic solution for the second type. This indicates that the present constraints of zero equivalent transverse displacement and rotation angle for a clamped end in (25) are looser than the first type of clamped end in [Timoshenko and Goodier 1970] but a little stiffer that the second type. For more slender beams, the differences between the three types of end constraints for clamped ends are small, and, hence, the results are almost identical, as can be seen in Figure 2, bottom. 

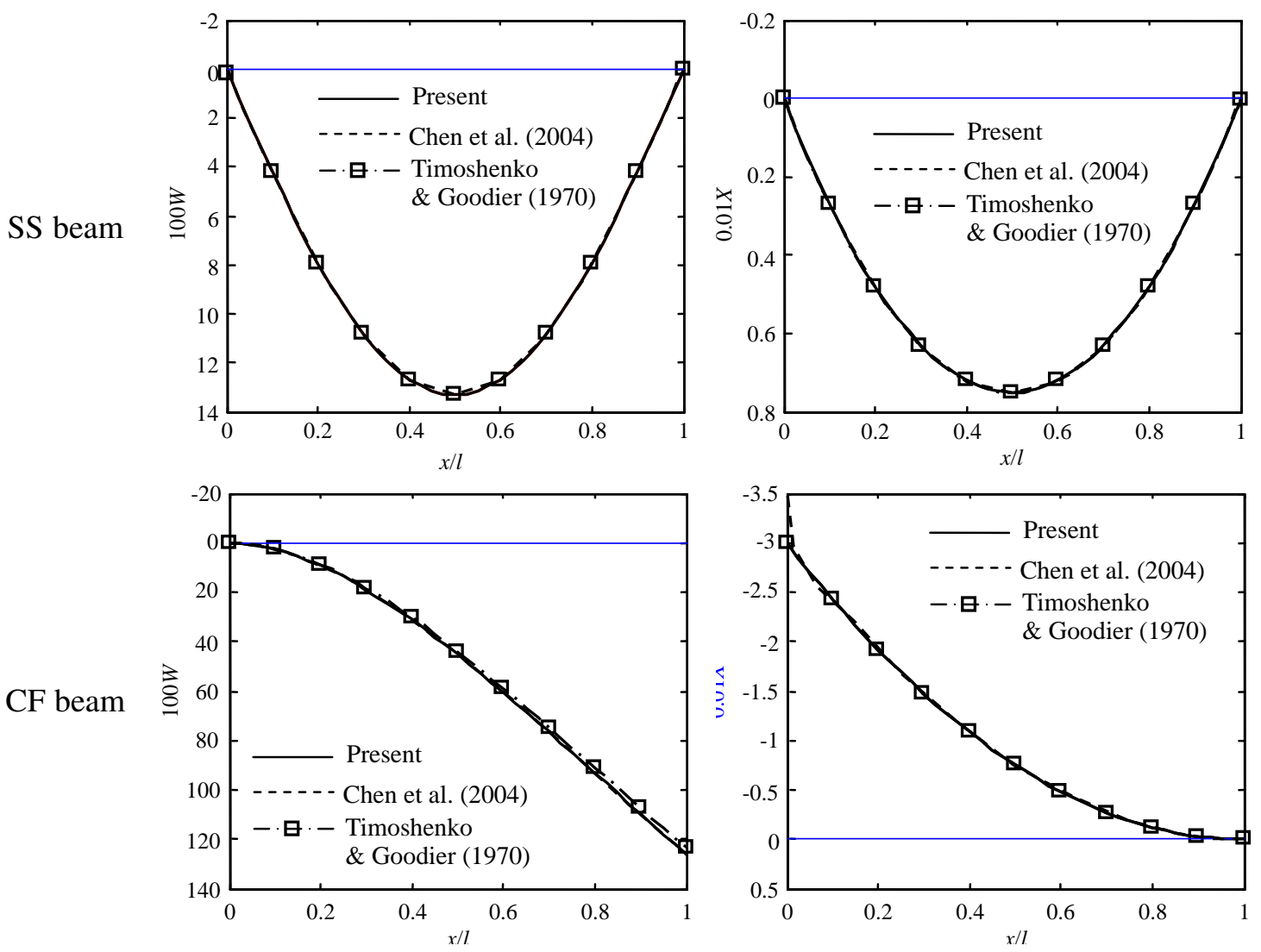

Figure 3. Deflection curves $W(x, 0)$ and longitudinal axial normal stresses $X(x, h)$ for SS and CF slender beams $(2 h / l=1 / 10)$ without elastic foundations $\left(K_{w}=K_{p}=0\right)$.

Figure 3 shows the longitudinal distributions of the transverse displacement $W(x, 0)$ at the neutral line and axial normal stress $X(x, h)$ at the lower surface of the SS and CF beams $(2 h / l=10)$. The Saint-Venant solutions of [Timoshenko and Goodier 1970] and the semianalytic elasticity solutions of [Chen et al. 2004] are also shown for comparison. As can be seen, the accuracy of the present method is again validated for slender beams.

Bending of beams on elastic foundations. We now assess the correctness of the symplectic formulation for beams resting on elastic foundations by comparison with the numerical results from the literature. Table 1 presents the nondimensional midspan deflection $W(0.5 l, 0)$ for uniformly loaded SS and CC beams on an elastic foundation with different values of $K_{w}$ and $K_{p}$. The semianalytic and exact solutions of [Chen et al. 2004], based on two-dimensional elasticity theory, are also listed for comparison. The results presented here, for all beams in consideration, compare well to the semianalytic results. A careful comparison shows that, for a moderately thick SS beam $(2 h / l=1 / 5)$, the present results agree better with the exact solutions than with the semianalytic solutions.

Figure 4 plots the deflection $W(x, 0)$ at the midplane and the axial normal stresses $X(x, h)$ at the bottom surface of a cantilever $(\mathrm{CF})$ beam on an elastic foundation with different values of $K_{w}\left(K_{p}=10\right)$. 


\begin{tabular}{|c|c|c|c|c|c|c|c|}
\hline & \multirow[b]{2}{*}{$K_{w}$} & \multirow[b]{2}{*}{$K_{p}$} & \multicolumn{3}{|c|}{ simply supported } & \multicolumn{2}{|c|}{ clamped-clamped } \\
\hline & & & present & semiana. & exact & present & semiana. \\
\hline \multirow{9}{*}{$\begin{array}{l}\underset{n}{=} \\
\frac{n}{\sqrt{n}} \\
\frac{\sqrt{2}}{=}\end{array}$} & & 0 & 1.31528 & 1.315277 & 1.315271 & 0.27500 & 0.27493 \\
\hline & 0 & 10 & 0.64831 & 0.648347 & 0.648299 & 0.21902 & 0.21893 \\
\hline & & 25 & 0.36736 & 0.367416 & 0.367353 & 0.16820 & 0.16811 \\
\hline & & 0 & 1.19135 & 1.191402 & 1.191335 & 0.26925 & 0.26921 \\
\hline & 10 & 10 & 0.61650 & 0.616562 & 0.616485 & 0.21533 & 0.21526 \\
\hline & & 25 & 0.35685 & 0.356923 & 0.356843 & 0.16600 & 0.16593 \\
\hline & & 0 & 0.64344 & 0.643767 & 0.643428 & 0.22653 & 0.22662 \\
\hline & 100 & 10 & 0.42717 & 0.427409 & 0.427156 & 0.18697 & 0.18701 \\
\hline & & 25 & 0.28361 & 0.283799 & 0.283603 & 0.14851 & 0.14853 \\
\hline \multirow{9}{*}{$\begin{array}{l}\frac{n}{2} \\
\frac{\pi}{\sqrt{2}}\end{array}$} & & 0 & 1.42083 & 1.420261 & 1.420243 & 0.39170 & 0.38814 \\
\hline & 0 & 10 & 0.67565 & 0.678202 & 0.674505 & 0.29722 & 0.29426 \\
\hline & & 25 & 0.37711 & 0.381703 & 0.376671 & 0.21910 & 0.21760 \\
\hline & & 0 & 1.27829 & 1.282598 & 1.277311 & 0.38014 & 0.37817 \\
\hline & 10 & 10 & 0.64134 & 0.646391 & 0.640247 & 0.29045 & 0.28874 \\
\hline & & 25 & 0.36608 & 0.372064 & 0.365680 & 0.21536 & 0.21478 \\
\hline & & 0 & 0.66969 & 0.696100 & 0.668478 & 0.29999 & 0.30908 \\
\hline & 100 & 10 & 0.43944 & 0.459267 & 0.438808 & 0.24086 & 0.24823 \\
\hline & & 25 & 0.28957 & 0.305161 & 0.289436 & 0.18662 & 0.19299 \\
\hline
\end{tabular}

Table 1. Midspan deflection $W(0.5 l, 0)$ of uniformly loaded SS and CC beams.
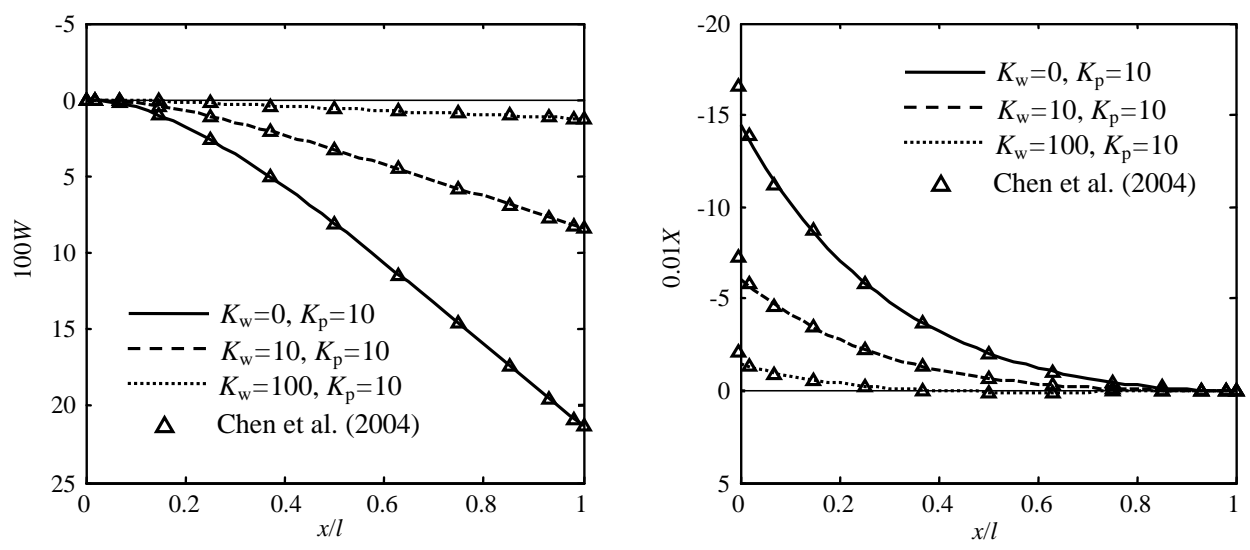

Figure 4. Longitudinal distributions of the deflection $W(x, 0)$ and axial normal stress $X(x, h)$ of a cantilever $(\mathrm{CF})$ beam on an elastic foundation $(2 h / l=1 / 15)$.

For numerical comparison, the semianalytic method in [Chen et al. 2004], based on two-dimensional elasticity theory, is applied here to calculate the semianalytic solutions, as denoted by the upward triangle markers in Figure 4. Excellent agreement is once again observed, further demonstrating the rationality and accuracy of the present symplectic method. 


\section{Conclusions}

The symplectic elasticity approach, which has been widely used in theoretical physics, is applied in this paper to derive analytic elasticity solutions for beams resting on Pasternak foundations. The Legendre transformation is adopted to transform the Lagrangian system, based on the minimum potential energy principle, to the Hamiltonian symplectic dual system. An eigenvalue problem with respect to the transverse cross section is, then, solved using the method of separation of variables. Saint-Venant solutions to the problem are obtained by considering the eigensolutions of zero eigenvalue. The effects of applied loading conditions and elastic foundations are treated as the boundary conditions. Together with the end constraints, the problem is formulated using the Hamiltonian variational principle.

Analytic Saint-Venant solutions for beams with different end constraints and on Pasternak foundations are presented. A comparison of the present results with those available in the literature are conducted, and, hence, the applicability and efficiency of the present symplectic approach for slender beams on elastic foundations are validated. It should be emphasized that the symplectic approach can be applied to study arbitrarily thick beams if nonzero eigenvalues are considered in the solution expansion, which will be a subject to be explored in the future.

\section{Appendix: Expressions of the matrix $D$ and vectors $p$ and $c$}

(Recall that $\boldsymbol{D} \boldsymbol{c}=\boldsymbol{p}$. See page 1747 for discussion. Except for the case $k_{w}=k_{p}=0$, we give $\boldsymbol{D}$ and $\boldsymbol{p}$, from which $c$ can be derived.)

Case 1: $k_{w} \neq 0$

CC beams:

$\boldsymbol{D}=\left[\begin{array}{cccc}\tilde{E}-\lambda M_{1} & \tilde{E}-\lambda M_{1} & \tilde{E}-\lambda M_{2} & \tilde{E}-\lambda M_{2} \\ \left(\tilde{E}+\lambda M_{1}\right) r_{1} & -\left(\tilde{E}+\lambda M_{1}\right) r_{1} & \left(\tilde{E}+\lambda M_{2}\right) r_{2} & -\left(\tilde{E}+\lambda M_{2}\right) r_{2} \\ \left(\tilde{E}-\lambda M_{1}\right) e^{r_{1} l} & \left(\tilde{E}-\lambda M_{1}\right) e^{-r_{1} l} & \left(\tilde{E}-\lambda M_{2}\right) e^{r_{2} l} & \left(\tilde{E}-\lambda M_{2}\right) e^{-r_{2} l} \\ \left(\tilde{E}+\lambda M_{1}\right) r_{1} e^{r_{1} l} & -\left(\tilde{E}+\lambda M_{1}\right) r_{1} e^{-r_{1} l} & \left(\tilde{E}+\lambda M_{2}\right) r_{2} e^{r_{2} l} & -\left(\tilde{E}+\lambda M_{2}\right) r_{2} e^{-r_{2} l}\end{array}\right], \quad \boldsymbol{p}=-\frac{\tilde{E} q_{0}}{k_{w}}\left\{\begin{array}{c}1 \\ 0 \\ 1 \\ 0\end{array}\right\}$.

SS beams:

$$
\boldsymbol{D}=\left[\begin{array}{cccc}
1 & 1 & 1 & 1 \\
M_{1} & M_{1} & M_{2} & M_{2} \\
e^{r_{1} l} & e^{-r_{1} l} & e^{r_{2} l} & e^{-r_{2} l} \\
M_{1} e^{r_{1} l} & M_{1} e^{-r_{1} l} & M_{2} e^{r_{2} l} & M_{2} e^{-r_{2} l}
\end{array}\right], \quad \boldsymbol{p}=-\frac{q_{0}}{k_{w}}\left\{\begin{array}{l}
1 \\
0 \\
1 \\
0
\end{array}\right\}
$$

CF beams:

$$
\boldsymbol{D}=\left[\begin{array}{cccc}
\tilde{E}-\lambda M_{1} & \tilde{E}-\lambda M_{1} & \tilde{E}-\lambda M_{2} & \tilde{E}-\lambda M_{2} \\
\left(\tilde{E}+\lambda M_{1}\right) r_{1} & -\left(\tilde{E}+\lambda M_{1}\right) r_{1} & \left(\tilde{E}+\lambda M_{2}\right) r_{2} & -\left(\tilde{E}+\lambda M_{2}\right) r_{2} \\
M_{1} e^{r_{1} l} & M_{1} e^{-r_{1} l} & M_{2} e^{r_{2} l} & M_{2} e^{-r_{2} l} \\
M_{1} r_{1} e^{r_{1} l} & -M_{1} r_{1} e^{-r_{1} l} & M_{2} r_{2} e^{r_{2} l} & -M_{2} r_{2} e^{-r_{2} l}
\end{array}\right], \quad \boldsymbol{p}=-\frac{\tilde{E} q_{0}}{k_{w}}\left\{\begin{array}{l}
1 \\
0 \\
0 \\
0
\end{array}\right\} .
$$


Case 2: $k_{w}=0, k_{p} \neq 0$

CC beams:

$$
\boldsymbol{D}=\left[\begin{array}{cccc}
\tilde{E}-\lambda M_{1} & \tilde{E}-\lambda M_{1} & \tilde{E} & 0 \\
\left(\tilde{E}+\lambda M_{1}\right) r_{1} & -\left(\tilde{E}+\lambda M_{1}\right) r_{1} & 0 & \tilde{E} \\
\left(\tilde{E}-\lambda M_{1}\right) e^{r_{1} l} & \left(\tilde{E}-\lambda M_{1}\right) e^{-r_{1} l} & \tilde{E} & \tilde{E} l \\
\left(\tilde{E}+\lambda M_{1}\right) r_{1} e^{r_{1} l} & -\left(\tilde{E}+\lambda M_{1}\right) r_{1} e^{-r_{1} l} & 0 & \tilde{E}
\end{array}\right], \quad \boldsymbol{p}=\frac{q_{0}}{k_{p}}\left\{\begin{array}{c}
-\lambda \hat{E} \\
0 \\
\frac{1}{2} \tilde{E} l^{2}-\lambda \hat{E} \\
\tilde{E} l
\end{array}\right\}
$$

where $\hat{E}=\bar{E}-0.4 k_{p} h^{2}$.

SS beams:

$$
\boldsymbol{D}=\left[\begin{array}{cccc}
1 & 1 & 1 & 0 \\
M_{1} & M_{1} & 0 & 0 \\
e^{r_{1} l} & e^{-r_{1} l} & 1 & l \\
M_{1} e^{r_{1} l} & M_{1} e^{-r_{1} l} & 0 & 0
\end{array}\right], \quad \boldsymbol{p}=\frac{q_{0}}{k_{p}}\left\{\begin{array}{c}
0 \\
\hat{E} \\
l^{2} / 2 \\
\hat{E}
\end{array}\right\}
$$

CF beams:

$$
\boldsymbol{D}=\left[\begin{array}{cccc}
\tilde{E}-\lambda M_{1} & \tilde{E}-\lambda M_{1} & \tilde{E} & 0 \\
\left(\tilde{E}+\lambda M_{1}\right) r_{1} & -\left(\tilde{E}+\lambda M_{1}\right) r_{1} & 0 & \tilde{E} \\
M_{1} e^{r_{1} l} & M_{1} e^{-r_{1} l} & 0 & 0 \\
M_{1} r_{1} e^{r_{1} l} & -M_{1} r_{1} e^{-r_{1} l} & 0 & 0
\end{array}\right], \quad \boldsymbol{p}=\frac{q_{0}}{k_{p}}\left\{\begin{array}{c}
-\lambda \hat{E} \\
0 \\
\hat{E} \\
0
\end{array}\right\}
$$

Case 3: $k_{w}=0$ and $k_{p}=0$

$\mathrm{CC}$ beams:

$$
\begin{aligned}
& a_{3}=\frac{q_{0}}{24 E I}(x-l)^{2} x^{2}-\frac{(1+v) q_{0}}{10 E A}\left(6 x^{2}-6 l x-l^{2}\right)-\frac{6 v(1+v) \lambda q_{0}}{5 E A}, \\
& a_{4}=\frac{3(1+v) q_{0}}{5 E A}(l-2 x)+\frac{q_{0}}{12 E I}(2 x-l)(x-l) x, \\
& a_{5}=-\frac{6 v(1+v) q_{0}}{5 E A}+\frac{q_{0}}{12 E I}\left(6 x^{2}-6 l x+l^{2}\right), \\
& a_{6}=\frac{q_{0}}{2 E I}(2 x-l),
\end{aligned}
$$

SS beams:

$$
\begin{aligned}
& a_{3}=\frac{3 q_{0}}{5 E A}(l-x) x+\frac{q_{0}}{24 E I}\left[(l+x) l-x^{2}\right](l-x) x, \\
& a_{4}=\frac{3 q_{0}}{5 E A}(l-2 x)+\frac{q_{0}}{24 E I}\left(l^{3}-6 l x^{2}+4 x^{3}\right), \\
& a_{5}=\frac{q_{0}}{2 E I}(x-l) x, \\
& a_{6}=\frac{q_{0}}{2 E I}(2 x-l) .
\end{aligned}
$$




\section{CF beams:}

$$
\begin{aligned}
& a_{3}=\frac{3 q_{0}}{5 E A}\left[(1+v)(l+2 x) l-x^{2}\right]+\frac{q_{0}}{24 E I}\left(x^{2}-4 l x+6 l^{2}\right) x^{2}, \\
& a_{4}=\frac{6 q_{0}}{5 E A}[(1+v) l-x]+\frac{q_{0}}{6 E I}\left(x^{2}-3 l x+3 l^{2}\right) x, \\
& a_{5}=\frac{q_{0}}{2 E I}(x-l)^{2}, \\
& a_{6}=\frac{q_{0}}{E I}(x-l) .
\end{aligned}
$$

\section{References}

[Chen et al. 2004] W. Q. Chen, C. F. Lü, and Z. G. Bian, "A mixed method for bending and free vibration of beams resting on a Pasternak elastic foundation”, Appl. Math. Model. 28:10 (2004), 877-890.

[De Rosa and Maurizi 1998] M. A. De Rosa and M. J. Maurizi, "The influence of concentrated mass and Pasternak soil on the free vibrations of Euler beams - Exact solution", J. Sound Vib. 212:4 (1998), 573-581.

[Ding et al. 2005] H. J. Ding, D. J. Huang, and W. Q. Chen, "Analytical solution for fixed-fixed beam subjected to uniform load", J. Zhejiang Univ. SCI. 6A:8 (2005), 779-783.

[Franciosi and Masi 1993] C. Franciosi and A. Masi, "Free vibrations of foundation beams on 2-parameter elastic soil", Comput. Struct. 47:3 (1993), 419-426.

[Frostig et al. 1992] Y. Frostig, M. Baruch, O. Vilnay, and I. Sheinman, "Higher-order theory for sandwich-beam behavior with transversely flexible core", ASCE J. Eng. Mech. 118:5 (1992), 1026-1043.

[Lee et al. 2003] Y. Y. Lee, C. M. Wang, and S. Kitipornchai, "Vibration of Timoshenko beams with internal hinge", J. Eng. Mech. 129:3 (2003), 293-301.

[Leissa 1973] A. W. Leissa, “The free vibration of plates”, J. Sound Vib. 31:3 (1973), 257-293.

[Lim 1999] C. W. Lim, "Three-dimensional vibration analysis of a cantilevered parallelepiped: exact and approximate solutions", J. Acoust. Soc. Am. 106:6 (1999), 3375-3381.

[Lim et al. 2007] C. W. Lim, S. Cui, and W. A. Yao, "On new symplectic elasticity approach for exact bending solutions of rectangular thin plates with two opposite sides simply supported”, Int. J. Solids Struct. 44:16 (2007), 5369-5411.

[Lim et al. 2008] C. W. Lim, W. A. Yao, and S. Cui, "Benchmarks of analytical symplectic solutions for bending of cornersupported rectangular thin plates", IES J. Part A 1:3 (2008), 106-115.

[Matsunaga 2000] H. Matsunaga, "Vibration and stability of thick plates on elastic foundations", ASCE J. Eng. Mech. 126:1 (2000), 27-34.

[Pasternak 1954] P. L. Pasternak, On a new method of analysis of an elastic foundation by means of two foundation constants (in Russian), Gosudarstrennoe Izdatelstvo Literaturi po Stroitelstvu I Arkhitekture, Moscow, 1954.

[Reddy 1984] J. N. Reddy, “A simple higher-order theory for laminated composite plates”, ASME J. Appl. Mech. 51:4 (1984), 745-752.

[Sankar 2001] B. V. Sankar, "An elasticity solution for functionally graded beams", Compos. Sci. Technol. 61:5 (2001), 689696.

[Shirima and Giger 1992] L. M. Shirima and M. W. Giger, "Timoshenko beam element resting on two-parameter elastic foundation", ASCE J. Eng. Mech. 118:3 (1992), 280-295.

[Sullivan and Vanoene 1986] J. L. Sullivan and H. Vanoene, "An elasticity analysis for the generally and specially orthotropic beams subjected to concentrated loads", Compos. Sci. Technol. 27:3 (1986), 133-155.

[Timoshenko 1921] S. P. Timoshenko, "On the correction for shear of the differential equation for transverse vibrations of prismatic bars", Philos. Mag. 41:6 (1921), 744-746.

[Timoshenko and Goodier 1970] S. P. Timoshenko and J. N. Goodier, Theory of elasticity, McGraw-Hill, London, 1970. 
[Wang et al. 1998] C. M. Wang, K. Y. Lam, and X. Q. He, "Exact solutions for Timoshenko beams on elastic foundations using Green's functions”, Mech. Struct. Mach. 26:1 (1998), 101-113.

[Weyl 1939] H. Weyl, The classical groups, their invariants and representations, Princeton University Press, 1939.

[Whitney 1985] J. M. Whitney, "Elasticity analysis of orthotropic beams under concentrated loads", Compos. Sci. Technol. 22:3 (1985), 167-184.

[Yao et al. 2009] W. A. Yao, W. X. Zhong, and C. W. Lim, Symplectic elasticity, World Scientific, Singapore, 2009.

Received 15 Jan 2009. Revised 22 Jun 2009. Accepted 9 Jul 2009.

C. F. LÜ: lucf@zju.edu.cn

Department of Civil Engineering, Zhejiang University, Hangzhou 310058, China

C. W. LIM: bccwlim@cityu.edu.hk

Department of Building and Construction, City University of Hong Kong, Tat Chee Avenue, Kowloon, Hong Kong

W. A. YAO: ywa@dlut.edu.cn

State Key Laboratory of Structural Analysis for Industrial Equipment and Department of Engineering Mechanics, Dalian University of Technology, Dalian 116024, China 